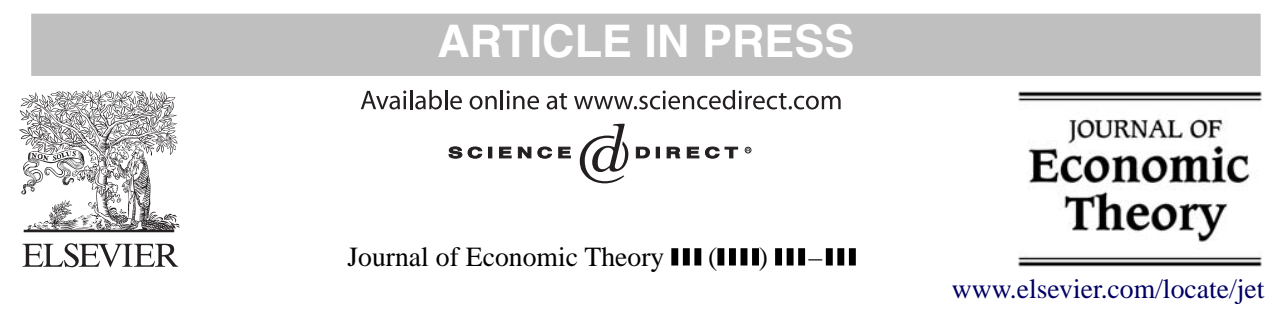

\title{
Contracts and externalities: How things fall apart
}

\author{
Garance Genicott ${ }^{\mathrm{a}, *}$, Debraj Ray ${ }^{\mathrm{b}}$ \\ ${ }^{a}$ Georgetown University, Washington, DC 20057-1036, USA \\ ${ }^{\mathrm{b}}$ New York University and Instituto de Análisis Económico (CSIC), USA
}

Received 23 August 2004; final version received 30 June 2005

\begin{abstract}
A single principal interacts with several agents, offering them contracts. The crucial assumption of this paper is that the outside-option payoffs of the agents depend positively on how many uncontracted or "free" agents there are. We study how such a principal, unwelcome though he may be, approaches the problem of contract provision to agents when coordination failure among the latter group is explicitly ruled out. Two variants are considered. When the principal cannot re-approach agents, there is a unique equilibrium, in which contract provision is split up into two phases. In phase 1, simultaneous offers at good (though varying) terms are made to a number of agents. In phase 2 , offers must be made sequentially, and their values are "discontinuously" lower: they are close to the very lowest of all the outside options. When the principal can repeatedly approach the same agent, there is a multiplicity of equilibria. In some of these, the agents have the power to force delay. They can hold off the principal's overtures temporarily, but they must succumb in finite time. In both models, despite being able to coordinate their actions, agents cannot resist an "invasion" by the principal and hold to their best payoff. It is in this sense that "things [eventually] fall apart".
\end{abstract}

(c) 2005 Elsevier Inc. All rights reserved.

JEL classification: D0; C7; L1; 017

Keywords: Multilateral externalities; Bilateral contracting; Coordination game; Exploitation; Delays

\section{Introduction}

A single principal interacts with several agents, offering them contracts. Our specification of a contract is reduced-form in the extreme: it is the offer of a payoff $c$ to the agent, with

\footnotetext{
* Corresponding author.

E-mail addresses: gg58@georgetown.edu (G. Genicot), debraj.ray@nyu.edu (D. Ray).
} 
consequent payoff $\pi(c)$ to the principal, where $\pi$ is some decreasing function. The crucial assumption of this paper is that the outside-option payoffs of the agents depend positively on how many "free agents" there are (these are agents who are not under contract). In short, the positive externalities imply that the agents are better off not having the principal around.

In this paper, we study how such a principal, unwelcome though he may be, approaches the problem of contract provision to agents. At the outset, however, we rule out two possible avenues along which the principal can make substantial inroads.

First, it is trivial to generate equilibria which rely on coordination failure among the agents, and which yield large profit to the principal. For instance, consider the value of the outside option when there is only a single free agent. This is the lowest possible value of the outside option. It is possible for the principal to simultaneously offer contracts to all the agents, yielding a payoff equal to the value of this option. If all agents believe that other agents will accept this offer, it is obviously a best response to accept. But such dire outcomes for the agents rely on an extreme form of coordination failure. They may happen, but in part because of the specific examples we have in mind, and in part because there is not much else to say about such equilibria, we are not interested in these situations. We explicitly refine such equilibria away by assuming that agents are always able to coordinate their actions.

Second, the principal may be able to offer "multilateral" contracts, the terms of whichas far as an individual is concerned-explicitly rely on the number of other individuals accepting contracts. It is easy enough to show that such contracts can effectively create prisoners' dilemmas among the agents, sliding them into the acceptance of low-payoff outcomes even in the absence of coordination failure. In keeping with a large literature on this subject (see below), we rule out such contracts. For reasons of enforceability, law, or custom, we assume that all contracts must be strictly bilateral.

The purpose of this paper is to show that even in the absence of these two avenues of potential domination by the principal, agents must "eventually" succumb to the contracts offered by the principal and at inferior terms, no matter which equilibrium we study (there may be more than one) and despite the existence of perfect coordination among the agents. What is of particular interest is the form this eventual takeover assumes. To describe it, we study two models.

In the first model, the principal can make contractual offers to agents, but cannot return to an agent who has refused him to make further offers. In the second model, the principal can return to an agent as often as he wishes. In both variants, time plays an explicit role; indeed, the dynamic nature of contractual offers is crucial to the results we obtain.

In the first specification, we show that there is a unique perfect equilibrium satisfying the agent-coordination criterion. In this equilibrium, the principal will generally split contract provision up into two phases. In phase 1, which we call the temptation phase, he makes simultaneous offers to a number of agents. [The offers are not the same, though.] In phase 2, which we call the exploitation phase, offers must be made sequentially, and their values are "discontinuously" lower: in fact, their values are close to the very lowest of all the outside options. ${ }^{1}$

\footnotetext{
${ }^{1}$ The degree of this closeness depends on the discount factor.
} 
Thus - in model 1-a community of agents is invaded in two phases: some agents are bought, the rest are "exploited", in the sense that at the time of their contract, their outside option is strictly higher than what they receive. Yet they cannot turn down the offer, because it is known by this stage that other agents must succumb to the sequential onslaught.

In the second model, there is a multiplicity of equilibria. In some of these equilibria all agents succumb to the principal right away. There are other equilibria in which the agents have the power to force delay. They can hold off the principal's overtures. But we show that they cannot hold the principal off forever; in every perfect equilibrium, agents must succumb in finite time. Indeed, even though the maximal delay becomes arbitrarily long as the discount factor approaches unity, the (discount-normalized) payoff of the agents must stay below and bounded away from the fully free reservation payoff.

It is in this sense that "things fall apart".

Multilateral externalities are, of course, widespread in practice and have received much attention in economics. The approach we take, in which the principal makes take-it-orleave-it offers to the agents, has much in common with Hart and Tirole [10], McAfee and Schwartz [17], Laffont et al. [15], Segal [22,23] and Moller [18]. The last two authors specifically address single-principal-many-agent problems with multilateral externalities. But the closest connection is to Segal and Whinston [24], published as an extended comment on Rasmusen et al. [20]. They consider a market in which an incumbent monopolist can offer exclusive contracts to some customers in order to discourage a potential competitor to enter. The "capture" of a certain number of agents prevents the rival from entering: the fixed costs are simply too high to deal with the few free agents that are left. In this scenario, the outside option of the agents takes a single step downwards after a certain "capture threshold" is passed. Our first model may be seen as a substantial extension and generalization of the Segal-Whinston paper. To begin with, we permit reservation utilities to "smoothly" decline as more and more agents come under the fold of the principal. Despite this "smoothness", the solution exhibits a "discontinuity" in the way that agents are treated-see the discussion of model 1 above. This does not contradict Segal and Whinston [24], of course, but there the "discontinuity" is already built in because of the special structure of their model: there is only one possible rival, and her exclusion brings about a jump in the outside options of the agents.

Next, our model instead has a completely open horizon, in which the game can-in principle-last forever, while payoffs are received in real time. Moreover, the timing is endogenous: agents can be approached simultaneously or sequentially, or in any combination of the two. ${ }^{2}$ And as we see, the results display a mixed timing structure; endogenously so. Some agents are approached simultaneously; others sequentially. We consider this dynamic (and the description of how it matters) to be a central methodological contribution of this paper.

An amplified precursor to [24] is Segal and Whinston's working paper, [25]. There, they also study a parallel to our model 2 , in which agents can be re-approached by the principal. They continue to assume that there are only a fixed number of stages. In this case our extended and more general scenario does not support the findings of their

\footnotetext{
${ }^{2}$ Segal and Whinston assume that agents must be approached one by one.
} 
particular model. With a fully dynamic, open-ended structure in which the principal cannot commit to never approach an agent to whom an offer has already been made, agents acquire considerably greater power, for now they can "punish" the principal along certain subgames. In contrast, a model with a fixed number of stages removes these agent capabilities by assumption, and indeed, as Segal and Whinston observe, makes exploitation easier. In our setting, the opposite is true. Yet we show that it cannot vanish entirely. Indeed, we show that the principal must finally "tie up" each agent in every equilibrium, though there may be delays that vary depending on the particular equilibrium or the discount factor.

It should also be noted that apart from the differences in generality and our methodological focus on endogenous timing, as well as the possible differences in the results (in model 2), the framework here is capable of application to a number of scenarios, and not just the industrial organization setting. To emphasize this point we provide several examples in Section 2, some of which originally motivated our research.

The rest of the paper is organized as follows: Section 3 introduces the dynamic model. Sections 4 and 5, analyze the equilibria of the two variants introduced above. Section 6 concludes. All proofs are relegated to Section 7.

\section{Examples}

There are, of course, several examples that fit into the general category of interest in this paper. We omit the obvious applications to network externalities in industrial organization and mention other examples.

\subsection{Bonded labor}

This example is in the spirit of Genicot [7]. A community of peasants supply labor inelastically over slack and peak seasons. Wages are low in the slack season and high in the peak season, and peasants seek to smooth consumption. There are two sources of credit: a risk-neutral landlord-moneylender, and a competitive fringe of moneylenders who lend on a short-term basis. The latter have heterogeneous overhead costs, so that the extent of fringe entry in any period is determined by the potential mass of "free borrowers" in that period.

At each date, the landlord can either offer simple labor contracts to the peasants, leaving them free to borrow from her or someone else later, or offer them "bonded labor" contracts, which are exclusive and combine labor, wage and credit specifications.

The more peasants sign a bonded contract the fewer the alternative credit sources that will be active. This lowers the outside options (and therefore the reservation payoffs) of a free borrower.

As a variation on this example, consider a single-period version with stochastic shocks to individual wages or employment status, workers could form an mutual "insurance fund" to protect one another. However, the greater the number of workers who enter into fixedwage contracts with the landlord, the thinner the supply of "free" workers and therefore the insurance fund. Once again, reservation payoffs exhibit positive externalities. 


\subsection{Working hours}

Consider a region with a number of residents, each endowed with time that is divided between work and leisure. Assume that the leisure activities of different residents are complements. There are several reasons why one might expect this. Not only do many people prefer spending their time off with their family and friends, but in addition, the availability of leisure activities (goods and services that are leisure-oriented) generally depends on the size of the market for it. [Consider, for instance, the decidedly poor (or perhaps one should say, even poorer) quality of TV programs during weekdays, or the smaller number of plays or concerts during the work week. See Makowski [16] for a model of the economy with an endogenous commodity space.] In brief, it is reasonable to suppose a reduced-form in which leisure time is less pleasurable when everybody else is working.

Now suppose that workers can either work full-time for a monopsonistic employer (and enjoy little or no leisure), or work part-time and enjoy substantial leisure. If a worker accepts a full-time offer, he inflicts an externality on his fellow-residents: the leisure-oriented community shrinks. As in the previous example, the worker's outside option is better when there are more "free workers" around.

\subsection{Bribing a committee}

Consider a group of $n$ agents, a committee or a group of voters, that has to ratify or reject a proposed law by majority voting. These voters are potentially under the influence of a lobbyist (see [4]). Assume that the agents experience an i.i.d. random loss if the legislation were to pass. If the expected loss is negative the legislation is likely to fail. Now imagine that, before voting takes place, a lobbyist attempts to bribe the committee members to get the project to pass. It is easy to see that the reservation payoff to an agent declines in the number of individuals who have effectively been bribed.

Two eighteenth century examples illustrate well this situation. Balen [1] recounts how the House of Commons and the Lords was initially clearly opposed to a bill allowing the South Sea Company to exchange the public's shares in the English public debt into its own shares. By bribing enough members, the South Sea Company got both Houses to pass the bill in 1720. In another example, Alexander Spotswood-lieutenant governor of Virginia in 1713 - wanted to pass a highly unpopular bill establishing a state monopoly on the shipment of the state's tobacco. Out of the 47-member House of Burgesses, 25 of them and the close relatives of 4 others received coveted positions as inspector for the shipment of tobacco. Bribed burgesses passed the Spotswood's bill (see [19]).

\subsection{Housing zones}

Consider a situation in which a real-estate developer plans to buy a green area to build houses. This green space is collectively owned by several individuals. The developer plans to convert each plot into an overcrowded housing complex, an outcome that none of the individuals prefers. Therefore, the larger the number of plots acquired by the developer, the lower is the reservation value of those individuals who still own their plots. 


\subsection{Takeovers}

A raider attempts to take over a company. Grossman and Hart [9] suggest that, after a takeover, a dilution of post-takeover value of each non-tendered share would occur. Examples of such dilution consist in large salary or option agreements by the raider, or sale of the target's assets or output to another company owned by the bidder. Such dilution may reduce the value of non-tendered shares to below its original value in the absence of takeover. The reservation utilities of the agents correspond to the original value of the shares as long less than $50 \%$ of the shares have been tendered to the raider. As soon as the raider has $50 \%$ of the shares, and thereby takes over the firm, the values of the non-tendered shares drops to its diluted level.

\section{Contracts with externalities}

A principal makes binding, bilateral, contractual offers to some or all of $n$ agents. We look at reduced-form versions of contracts: an offer of a payoff $c$ is made to an agent, and this payoff is received every period for life. The payoff to the principal from a contract $c$ is $\pi(c)$, where $\pi$ is a continuous, decreasing function. A contract vector, or simply an offer, is just a list of agent payoffs $\mathbf{c}$ listed in non-decreasing order: $c_{k} \leqslant c_{k+1}$. As for which agents are actually receiving the contracts, the context should make this amply clear.

At any date, an agent is either "contracted" (by the principal) or "free". In the latter case, she has either turned down all offers from the principal, or has never received one. A "free agent" receives a one-period payoff $r_{k}$, where $k$ is the number of free agents (counting herself) at that date. The $r_{k}$ 's are parameters of the model, representing (one-shot) reservation payoffs when there are $k$ free agents. Our basic assumption, maintained throughout the paper, is

[A.1] Positive spillovers. $r_{k}<r_{k+1}$ for all $k=1, \ldots, n-1$.

At any date $t \geqslant 1$, a history - call it $h_{t}$-is a complete list of all that has transpired up to date $t-1$ : agents approached, offers made, acceptances etc. [Use the convention that $h(0)$ is some arbitrary singleton.] Let $N\left(h_{t}\right)$ be the set of available agents given history $h_{t}$. These are free agents who are available to receive an offer; more on this specification below.

The game proceeds as follows. At each date $t$ and for each history $h_{t}$, the principal selects a subset $S_{t} \subseteq N\left(h_{t}\right)$ of available agents and announces a personalized or anonymous offer (vector) c to them. [Note: $S_{t}$ may be empty, so that not making any offer is permitted.] All agents in $S_{t}$ simultaneously and publicly accept or reject their component of the offer. Then the history is appropriately updated and so is the set of available agents. The process repeats itself at date $t+1$, and is only declared to end when the set of available agents is empty.

Lifetime payoffs are received as the sum of discounted one-shot payoffs. Once contracted, an agent with offer $c$ simply receives $c$ every period for life, while a free agent obtains the (possibly changing) reservation payoffs. The principal makes no money from free agents, and $\pi(c)$ every period from an agent contracted at $c$. We assume that the agents and the principal discount the future using a common discount factor $\delta \in(0,1)$. We normalize 
lifetime payoffs by multiplying by $1-\delta$. By formally specifying strategies for the principal and agents, we may define subgame-perfect equilibrium in the usual way.

Observe that $r_{n}$ may be viewed as the "fully free" payoff, obtained when no contracts have been signed. On the other hand, $r_{1}$ may be viewed as the "fully bonded" payoff: it is the outside option available to a free agent when there are no other free agents around.

Now, it is always possible for the principal to implement the fully bonded contract if we allow for coordination failure across agents: all she does is make the offer $\left(r_{1}, r_{1}, \ldots, r_{1}\right)$ to all the agents at date 0 . It is an equilibrium to accept. Of course, it is an equilibrium which the agents could have coordinated their way out of: saying "no" is also sustainable as a best response. In this paper, we not only allow for such coordination, we insist on it. [In any case, if one wishes to seriously entertain coordination failure, its consequences are obvious.] Especially given the small or rural communities we have in mind, we would like to imagine communication as taking place easily among the agents. An agent who is about to accept an offer has no incentive to state otherwise, while an agent who plans on rejecting has all the interest in revealing his intention. Therefore, we impose throughout the following assumption:

[A.2] Agent coordination. Restrict attention to perfect equilibria with the following property: there is no date and no subset of agents who have received offers at that date, who can change their responses and all be strictly better off, with the additional property that the changed responses are also individual best responses, given the equilibrium continuation from that point on. ${ }^{3}$

Just in case it is not obvious, we stress that [A.2] only permits agents to coordinate their moves at particular stages. In particular, [A.2] in no way selects-among possibly many equilibria - the equilibrium preferred by the agents. We also note that coordination necessitates that the "coordinated responses" must be individually incentive-compatible; in particular, we assume that agents do not have a commitment device that allows them to make binding transfers to one another conditional on taking certain actions. ${ }^{4}$

It should be noted that agent coordination carries little bite if the principal is permitted to offer contracts that are contingent on the simultaneous acceptance-rejection decisions of other players. If such contracts are permitted, the principal can create "prisoners' dilemmas" for the agents and get them all to accept the lowest possible price $r_{1}$. In line with most of the literature, we rule out such contracts.

In what follows, we study two leading cases. In the first case, discussed in Section 4, each agent can receive at most a single offer. Formally, $N\left(h_{t+1}\right)=N\left(h_{t}\right) \backslash S_{t}$. In the second leading case, studied in Section 5, all the free agents at any date are up for grabs: $N\left(h_{t+1}\right)$ is just $N\left(h_{t}\right)$ less the set of agents who accepted an offer in period $t$.

\footnotetext{
${ }^{3}$ In this specific model, agent coordination may be viewed as a coalition-proofness requirement applied to the set of agents. Because of the specific structure, the nested deviations embodied in the definition of coalition-proof equilibrium do not need to be invoked. Because a satisfactory definition of coalition-proofness does not exist for infinite games, we do not feel it worthwhile to state this connection more formally.

${ }^{4}$ While such commitment power does not necessarily lead to efficiency—see, e.g., Ray and Vohra [21] and Gomes and Jehiel [8], it can be shown that in this context efficiency would, indeed, prevail and the principal would only be able to contract the agents at their fully free outside option.
} 


\section{The single-offer model}

In this section, it is assumed that the principal cannot make more than one offer to any agent. It will be useful to begin with a simple example.

Example 1. Suppose, to start with, that there are just two agents. Let $\delta=0.9, r_{1}=10$ and $r_{2}=20$, and suppose that $\pi(r)=25-r$. We solve this game "backwards". Suppose that there is only one available agent. If the other agent is free (he must have rejected a previous offer), then the principal will offer the available agent 20, and if the other agent is contracted, then the available agent will receive 10 .

Now study the game from its starting point. Define $\widetilde{r}_{2} \equiv(1-\delta) r_{2}+\delta r_{1}=11$, which is the stationary payoff that's equivalent to enjoying $r_{2}$ in one period and $r_{1}$ ever after. Given the calculations in the previous paragraph, it is easy to see that the principal will make an offer of 11 to a single agent, which must be accepted. Tacking on the second stage from the previous paragraph, the remaining agent will receive 10 in the second period. The overall (discount-normalized) payoff to the principal is $27.5(14+0.9 \times 15)$.

We can see that the principal cannot benefit from making simultaneous offers in the first period, even though he would like to do so because he discounts future payoffs. By the agent coordination criterion, the only payoff vector which he can implement is $(20,10)$, yielding him a (normalized) payoff of 20, lower than that from the staggered sequence in the previous paragraph.

Now add a third agent. Let $r_{1}$ and $r_{2}$ be as before, and set $r_{3}=28$. Once again, we solve this problem backwards. We already know what will happen if one of the agents is contracted and the other two are available; this is just the case we studied above. If, on the other hand, there are two agents available but the unavailable agent is free (he refused a previous offer), this induces a two agent problem with reservation values $\left(r_{1}^{\prime}, r_{2}^{\prime}\right)=\left(r_{2}, r_{3}\right)=$ $(20,28)$.

This subproblem is different from the one we studied before. If a single agent refuses an offer, the principal will not make an offer to the one remaining agent, because the agent's reservation value stands at 28 and the principal's contractual profits will be negative. Knowing this, if any single agent receives an offer, he will hold out for 28 , and the remaining agent will be paid 20. Now the principal will prefer to make a simultaneous offer with payoffs $(28,20)$, which is accepted in equilibrium.

It follows that the very first of our three agents can — through refusal—obtain a payoff of $\widetilde{r}_{3} \equiv(1-\delta) r_{3}+\delta r_{1}=11.8$. By making fully sequential offers, the principal implements the payoffs $(11.8,11,10)$ for the three agents. Once again, the coordination criterion assures us that he can do no better making a simultaneous offer.

Matters are different, however, if we change the value of $r_{3}$ to 31 and leave everything else unchanged. Now revisit the subproblem in which there are two available agents, and the third agent is free (but no longer available). It is now easy to see that the principal will make no offers at all. For the induced two-agent problem has reservation values $\left(r_{1}^{\prime}, r_{2}^{\prime}\right)=$ $\left(r_{2}, r_{3}\right)=(20,31)$, and there is no way the principal can turn a positive profit in this subgame. The third agent is now pivotal. By refusing, he can ensure that the principal goes no further. 
The only way for the principal to proceed in this case is to make an initial offer of $r_{3}=31$ to a single agent, which will be accepted. With this agent contracted and out of the way, the principal can now proceed to give the remaining agents 11 and 10 (sequentially). There is no harm in making the offer of 11 up front as well but the offer of 10 must wait a further period. This yields a payoff of $-6+14+\delta \times 15=21.5$. Again, it can be checked, using the coordination criterion, that no simultaneous offer will do better.

To summarize the discussion so far, we see that

(1) Either the third agent is "pivotal" in that his refusal sparks a market collapse, in which case he obtains "discontinuously more" than the other two agents; or

(2) The third agent's actions has no bearing on what happens next-future agents are always contracted-in which case he receives a low payoff.

The phrases "discontinuously more" and "low payoff" are best appreciated when the discount factor is close to one. The "low payoff" is then essentially $r_{1}$, while a "discontinuously higher" payoff entails receipt of some $r_{k}$, for $k>1$.

If the example so far has been absorbed, consider the addition of a fourth player. We will study the pivotal three-player subcase: hence retain $\left(r_{1}, r_{2}, r_{3}\right)=(10,20,31)$. Let us suppose that $r_{4}=35$. Consider again an offer to a single agent (call him the fourth agent). If he refuses the offer, we induce the three-agent game with reservation values $\left(r_{1}^{\prime}, r_{2}^{\prime}, r_{3}^{\prime}\right)=\left(r_{2}, r_{3}, r_{4}\right)=(20,31,35)$. This game is way too costly for the principal, and no further offer will be made. The fourth player is therefore pivotal: his refusal will shut the game down. On the other hand, his acquiescence induces the three-agent game in which agent 3 is pivotal as well. It follows that both the third and fourth players need to be bought out at the high reservation values. This can be done sequentially but given discounting, the right way to do it is to make the discontinuously high simultaneous offer $\left(r_{3}, r_{4}\right)=(31,35)$ to any two of the players, and then follow through with sequential contracting using the low offers $(11,10)$. Once again, the sequential process can begin at date 0 as well. It is easy to check, using the coordination criterion, that no other offer sequence is better, and once again we have solved for the equilibrium.

The four-agent game yields a couple of fresh insights. If the third agent is pivotal conditional on the fourth agent's initial acquiescence, the fourth agent must be pivotal as well. For the third player's pivotality (conditional on the fourth agent's prior acquiescence) simply means that $\left(r_{3}, r_{2}\right)$ is an unprofitable 2-agent environment for the principal. Now if the fourth agent were to refuse an initial offer, this would induce the three-agent environment $\left(r_{4}, r_{3}, r_{2}\right)$. If the principal cannot turn a profit in the environment $\left(r_{3}, r_{2}\right)$, he cannot do so under $\left(r_{4}, r_{3}, r_{2}\right)$, which is even worse from his point of view. Therefore the fourth agent must be pivotal as well.

The example suggests, therefore, that

(3) The pivotality of a player in an environment in which all "previous" agents have accepted their offers implies the pivotality of these "earlier" agents in an analogous environment. But more is suggested. If all these pivotal players need to be bought at their reservation values, there is no sense in postponing the inevitable (recall that the overall profit of the principal is positive for there to be any play at all, and there is discounting). Therefore, 
(4) Pivotal agents are all made simultaneous (but unequal) offers at the high reservation values.

In contrast,

(5) The remaining non-pivotal players are offered low payoffs (approximately $r_{1}$ when $\delta \simeq 1$ ) and must be approached sequentially so as to avoid the use of the coordination criterion.

[Remember, in reading this, that all players are identical, so that by "pivotal players" we really mean pivotal player indices.]

Finally, it is easy to see in this four-agent example the total surplus is lower when agents enter into contracts with the principal, so that:

(6) The principal is able to sign up all agents even in situations where this is inefficient.

In what follows, we show that the insights of Example 1 can be formalized into a general proposition. This is a characterization of unique equilibrium, which obtains under the following convention: the principal will immediately make an offer and an agent will immediately accept an offer if they are indifferent between doing so and not doing so.

Proposition 1. There is $\hat{\delta} \in(0,1)$ such that for every $\delta \in(\hat{\delta}, 1)$, there exists an equilibrium satisfying [A.2], which is unique up to a permutation of the agents. Either the principal makes no offers at all, ${ }^{5}$ or the following is true: there is $m(\delta) \in\{1, \ldots, n\}$ such that for agent $j$ with $j \geqslant m(\delta)$, an offer of precisely $r_{j}$ is made in the very first period, date 0 . All these offers are accepted. Thereafter, agent $j<m(\delta)$ is made an offer $r_{j}^{\prime} \in\left[r_{1}, r_{1}(\delta)\right]$ at date $m(\delta)-j-1$, and these offers are accepted as well.

The index $m(\delta)$ is non-decreasing in $\delta$, and $r_{1}(\delta) \rightarrow r_{1}$ as $\delta \rightarrow 1$.

The proposition shows that there is a unique equilibrium path, which exhibits two distinct phases. Because all agents are identical, the uniqueness assertion is obviously subject to arbitrary renaming of the agents.

The first phase is the temptation phase. Agents with indices above $m(\delta)$ are pivotal. If they reject an offer, then in the resulting continuation subgame, the principal cannot make offers that are both acceptable and profitable. Hence, they receive relatively high and differentiated offers. By the agent coordination criterion, to prevent a subset of size $s$ of pivotal agents from rejecting her offer, the principal needs to offer at least $r_{m(\delta)+s}$ to one of these agents. This reasoning applied to subsets of all sizes between 1 and $n-m(\delta)+1$ determines the vector of offers. They must be bought outright, because of discounting.

In contrast, in the second phase, which we may call the exploitation phase, corresponds to the sequential "acquisition" of the remaining $m(\delta)-1$ agents starting in period 0 . They are made "low" offers that converge to $r_{1}$ as $\delta$ goes to one (these are analogous to the payoffs of 10, 11, and 11.8 in Example 1). This procedure must be sequential even though the principal is eager to complete the acquisitions (by discounting). Any form of simultaneity at this stage

\footnotetext{
${ }^{5}$ Equivalently, he makes offers that are unacceptable.
} 
would be blocked by the agent coordination criterion: the agents would benefit from a joint refusal. ${ }^{6}$

A contract may be called exploitative when a party uses its power to restrain the set of alternatives available to another party, so as the latter has no better choice than to agree upon a contract very advantageous to the first party (see Basu [3], Hirsheifer [11], Bardhan [2] and Genicot [7]). Here, the reason the principal is willing to incur losses in the temptation phase is because, by doing so, she lowers the reservation utility of the other agents and put them in a situation in which their best option is to accept very low offers. This is the very idea that characterizes the exploitation phase.

Note well the discontinuity between the two phases. Agent $m(\delta)$ receives $r_{m(\delta)}$. Agent $m(\delta)-1$ receives something close to $r_{1}$.

This result is closely related to Rasmusen et al. [20] as corrected by Segal and Whinston [24]. These two papers study a market in which a monopolist incumbent can offer exclusive contracts to customers in order to discourage potential entry. The reservation utility of the agents is a step function that jumps from 0 to a higher value at the minimum market size needed for the entrant to enter. So there is a specific number of agents that the monopolist needs to sign up in the first period in order to discourage entry. Assuming fully sequential offers, these papers unearth a similar pivotality idea: some agents receive the high value while the others receive 0 . Our results show that these type of discontinuity in the offers received by the agents is actually a general feature of all models with positive externalities in the reservation utility, even if the reservation utility increases smoothly with the number of agents. Moreover, by fully endogenizing the approach of the principal we show that her best policy is not fully sequential: some offers are made simultaneously while others are made sequentially.

Some easy generalizations of this result are available. For instance, we have assumed here that the principal's payoff function is linear in the number of agents who sign a contract. However, Proposition 6 would hold for any nonlinear payoff function as long as it is increasing in the number of bonded agents. Likewise, it is easy enough to extend the proposition to the case of heterogeneous agents. Heterogeneity would induce a specific order in which the principal approaches the agents. Two important factors in predicting the identity of the "early" agents would be the immediate profit an agent generates for the principal and the size of the externality a contracted agent has on other agents' reservation utilities. To our mind, however, the homogeneous case, while formally more special, is more compelling, as it illustrates how symmetry must be broken along the optimal path (for the principal). [With heterogeneities to begin with, such symmetry-breaking would be masked.]

Finally, note that our assumed resolution of indifference does matter in ruling out multiple equilibria (with distinct payoffs). As an example, take a two-agent game in which $\pi\left(r_{1}\right)>0$ and $\pi\left(r_{2}\right)=0$. Assume that agent 2 gets the first offer and agent 1 the second. If agent 1 accepts offers when indifferent, agent 2 is non-pivotal and will therefore accept an offer in

\footnotetext{
${ }^{6}$ Note that either phase may be empty but that once non-empty, the exploitation phase remains non-empty for all higher discount factors. Also note that the exploitation phase is also characterized by differentiated offers. For instance, the first person in that phase receives strictly more than $r_{1}$ (though close to it, as noted), while the last person receives precisely $r_{1}$. However, the differentiation of offers, while also an essential feature of this phase, is not as striking or important as in the temptation phase.
} 
$\left(r_{1}, r_{1}(\delta)\right)$. In contrast, if agent 1 rejects offers when indifferent, then 2 is pivotal and won't accept offers of less than $r_{2}$. This equilibrium exhibits a lower payoff for the principal. In a three-agent game, then, we can support payoffs to agent 3 that are lower than $r_{3}$ but larger than $r_{1}(\delta)$ simply by having agent 1 threaten the principal by resolving indifference in different ways, depending on history. This is a substantial departure, and would make a complete characterization of the equilibria hopeless.

\section{The model with multiple offers}

While the results of the previous section are methodologically striking in that they illustrate the necessity of unequal treatment, we must qualify the model. The main assumption made is that a principal can commit not to return to an agent who has rejected an offer. In many situations — and despite its obvious advantages to the principal—such a commitment technology may be unavailable. It is therefore imperative to consider the case of no commitment, in which the principal can make multiple offers to a single agent. Can agents now "resist" the principal's overtures?

It turns out that this model exhibits multiple equilibria. Nevertheless, we show that, under assumption [A.3] below, agent payoffs must be damaged in the presence of the principal, whether or not such an outcome is efficient for the economy as a whole. In some equilibria, agents temporarily resist the principal's offer and there are delays (which the agents like). The delays even become unboundedly large as the discount factor goes to one. Nevertheless, we show that the average payoff of agents must stay below and bounded away from their free payoff $r_{n}$, uniformly in the discount factor.

Consider the following scenario. At the start of any period, the principal makes contractual offers to some or all of the free agents at that date. Agents who accept an offer become "unfree" or "contracted" and must hold that contract for life. ${ }^{7}$ After these decisions are made, payoffs are received for that period. Contracted agents receive their contract payoffs. Each free agent receives an identical payoff $r_{k}$, where $k$ is the number of free agents at the end of the decision-making process. The very same agents constitute the starting set of free agents in the next period, and the process repeats itself. No more offers are made once the set of free agents is empty.

Throughout this section, the following assumption will be in force:

[A.3] $\sum_{i=1}^{n} \pi\left(r_{i}\right)>0$.

It will be clear from what follows that if this assumption was violated, no offer would be made. Therefore, we focus on situation where [A.3] holds. To establish our main results, it will be useful to study the worst and best equilibrium payoffs to the principal (in addition, these observations may be of some intrinsic interest). First, some definitions. Suppose that at any date a set of $m$ free agents are arrayed as $\{1, \ldots, m\}$. We shall refer to these indices as the names of agents. Next, look at the special package $\mathbf{r}$ made to all $m$ free agents,

\footnotetext{
${ }^{7}$ Later, we comment on contracts which can be ended after some finite duration. As far as our results are concerned, not much of substance changes.
} 
where $\mathbf{r}=\left(r_{1}, \ldots, r_{m}\right)$. We shall call this the standard offer (to $m$ free agents), where it is understood that the agent with name $i$ receives the offer $r_{i}$.

Next, define for any $m, \hat{r}_{m} \equiv(1-\delta) r_{m}+\delta r_{1}$. As interpretation, this would be the (normalized) lifetime payoff to a free agent when he spends one period of "freedom" with $m-1$ other free agents, and is then the only free agent for ever after. Now look at the special package $\hat{\mathbf{r}}$ made to all $m$ free agents, where $\hat{\mathbf{r}}=\left(\hat{r}_{1}, \ldots, \hat{r}_{m}\right)$. We shall call this the low offer (to $m$ free agents). Once again, it is understood that the agent with name $i$ receives the offer $\hat{r}_{i}$.

The following proposition pins down worst and best (discount-normalized) equilibrium payoffs to the principal.

Proposition 2. The principal's worst equilibrium payoff is precisely his payoff from the standard offer made to-and accepted by-all $n$ agents:

$$
\underline{P}(n)=\sum_{i=1}^{n} \pi\left(r_{i}\right),
$$

while his best equilibrium payoff arises from the low offer made to-and accepted by-all $n$ agents:

$$
\bar{P}(n)=\sum_{i=1}^{n} \pi\left(\hat{r}_{i}\right) .
$$

As discussed in the introduction, these results are in sharp contrast with models in which a sequential approach is exogenously assumed and where being able to re-approach the agents only helps the principal (see Segal and Whinston [25]).

The standard offer is similar to the divide-and-conquer offer that appears in the static context (see for instance $[12,23,24,26]$ ). Indeed, it is easy enough to see that the principal can guarantee at least $\sum_{i=1}^{n} \pi\left(r_{i}\right)$ in any equilibrium. [Simply make the standard offer to all agents plus a bit more, and they will all accept.] The difficulty is to show that the standard offer is actually an equilibrium outcome in a dynamic model. It is conceivable that the principal might be able to deviate from the standard offer (or its payoff-equivalent contractual offer) in ways that are beneficial to himself. This possibility pushes the equilibrium construction in a complicated direction. Various phases need to be constructed to support the original equilibrium payoff, and at each date several incentive constraints must be checked. Section 7 contains the details, which are not trivial.

The standard-offer equilibrium can be used to shore up other equilibria. In particular, we can use it to calculate the best equilibrium payoff to the principal. First, make the low offer $\hat{\mathbf{r}}$ to all free agents (under some naming). If any subgroup of $m$ agents rejects its component of this offer, rename the deviators so that the rejector with the "highest" name is now given the "lowest" name. Now start up the standard-offer equilibrium with this set of free agents. This ensures that the highest-named deviator (agent $m$ ) earns no more than

$$
(1-\delta) r_{m}+\delta r_{1}
$$

from her deviation. But she was offered $\hat{r}_{m}=(1-\delta) r_{m}+\delta r_{1}$ to start with, anyway. She therefore has no incentive to go along with this group deviation. We therefore have an 
equilibrium, which we shall refer to as the low-offer equilibrium. Notice that it satisfies the agent-coordination criterion. ${ }^{8}$

Indeed, it is precisely because of [A.2] that even better payoffs are unavailable to the principal, and why $\bar{P}$ as described in (2) is truly the best equilibrium payoff. To see this, it suffices to recall that $r_{1}$ is the worst possible continuation utility an agent can ever receive. Hence, to sign on any agent when $n$ agents are free, the principal has to make at least one offer that is better than $\hat{r}_{n}$ for the agent. Otherwise, the agents would profitably coordinate their way out of such an offer. Given this fact, the principal must make an offer of $\hat{r}_{n-1}$ or better to sign up another agent, whether in the current period or later. By repeating the argument for all remaining agents, we may conclude that the principal can earn no more than a payoff of $\bar{P}(n)=\sum_{i=1}^{n} \pi\left(\hat{r}_{i}\right)$, and this completes our intuitive discussion of Proposition 2.

We turn now to the main topic of interest: the extent to which the agents can hold on to their "best payoff" of $r_{n}$ against an "invasion" by the principal. To be sure, some agent might get $r_{n}$ (see, for instance, the standard-offer equilibrium). But we are interested in average agent payoffs, not the best payoff to some agent. To this question one might attempt to invoke the standard-offer equilibrium to provide a quick answer. Surely, if this is the worst equilibrium for the principal, it yields the best average payoff for the agents, which is still lower than $r_{n}$. However, the answer is not that simple, because the standard-offer equilibrium for the principal may not correspond to the one with the best average payoffs for the agents (see Example 2 below).

Indeed, equilibria with some initial delay are generally a good thing for the agents: each agent obtains the full payoff $r_{n}$ for each period in which no offers are made or accepted. So one measure of "agent resistance" is the length of a maximal delay equilibrium: the amount of time for which no offers are made (or accepted, if one is made). Proposition 3 below gives us a bound as an easy consequence of our worst and best equilibrium characterizations. ${ }^{9}$

\section{Proposition 3. An equilibrium with initial delay $T$ exists if and only if}

$$
T \leqslant \frac{\ln (\underline{P}(n))-\ln (\bar{P}(n))}{\ln \delta} .
$$

The arguments underlying this proposition are quite simple. To support the longest possible delay, the principal must receive her highest possible payoff at the end of the delay, while she must receive the worst possible payoff if she deviates. Hence, if $T$ is the size of the delay, we must have $\delta^{t} \bar{P}(n) \geqslant \underline{P}(n)$, which yields (3).

\footnotetext{
${ }^{8}$ The reader may be uncomfortable that agent $m$ is just indifferent to the deviation, while the others may strictly prefer the deviation. In that case, $\bar{P}$ as described in (2) may be thought out as the supremum equilibrium payoff, where the open-set nature of the weaker agent-coordination criterion prevents the supremum from being attained Either interpretation is fine with us.

${ }^{9}$ Cai [5] studies delays arising in a model with one principal and many agents but in a very different setting. The complementarities arise within the contract, not in the reservation utility. In Cai's model, the principal needs to sign all agents in order to get any revenue; this externality matters because the agents have some bargaining power. When the principal makes take-or-leave it offers in a perfect-information context, only externalities in the reservation utility matter.
} 
Delays arise in other models with multilateral externalities, though in very different settings. In Gale [6], the payoff from an irreversible investment depends on the number of investors. Hence, agents would like to coordinate the date of their investment, and there are multiple equilibria, some with delays. Jehiel and Moldovanu [13,14] show how delays can arise when an indivisible object is to be sold to one of several potential buyers, the seller is randomly matched with one buyer per period, and the identity of the final buyer affects the payoffs of her rivals.

Notice that less discounting is amenable to the creation of greater delay. This makes sense: the principal must be induced to accept the delay, instead of bailing out - say, with the use of the standard offer. Indeed, if $\delta$ is small enough no delay is possible, because $\underline{P}$ and $\bar{P}$ come arbitrarily close to each other, and in addition the principal is in a hurry to close deals as soon as possible. ${ }^{10}$ On the other hand, as $\delta$ approaches unity, it is easy to see from (3) that arbitrarily long delays can be sustained.

Proposition 3 can easily be used to construct an example in which agent average payoffs exceed those under the standard-offer equilibrium. This happens precisely when there is delay.

Example 2. Suppose that $\pi(r)=Y-r$ for some $Y>0$. We will show that if $\pi\left(r_{n}\right)=$ $Y-r_{n}<0$, then the best average payoff to an agent exceeds the average under the standardoffer equilibrium, which is $\left(\sum r_{i}\right) / n$.

Along the lines suggested by Proposition 3, construct an equilibrium in which nothing happens for the first $T$ periods, after which the principal initiates the low-offer equilibrium. Any deviations by the principal during this initial "quiet phase" is punished by reversion to the standard-offer equilibrium, so that the largest possible such $T$ is the largest integer that satisfies

$$
\delta^{T} \geqslant \frac{P}{\bar{P}}=\frac{Y-\left(\sum r_{i}\right) / n}{Y-\left(\sum \hat{r}_{i}\right) / n} .
$$

For the purposes of this example, we shall choose $\delta$ so that equality holds in the inequality above (i.e., so that integer problems in the choice of $T$ can be neglected)

$$
\delta^{T}=\frac{P}{\bar{P}}=\frac{Y-\left(\sum r_{i}\right) / n}{Y-\left(\sum \hat{r}_{i}\right) / n} .
$$

Such an equilibrium would yield an average agent payoff of

$$
a_{T}=\left(1-\delta^{T}\right) r_{n}+\delta^{T}\left(\sum \hat{r}_{i}\right) / n .
$$

Recalling that $\hat{r}_{i}=(1-\delta) r_{i}+\delta r_{1}$, it is possible to show that $a_{T}>\sum_{i} r_{i} / n$ if (and only if)

$$
\frac{1-\delta^{T}}{\delta^{T+1}}>\frac{\left(\sum_{i} r_{i}\right) / n-r_{1}}{r_{n}-\left(\sum_{i} r_{i}\right) / n}
$$

${ }^{10} \mathrm{~A}$ sufficient condition for there to be no delay in equilibrium is $\frac{\delta^{2}}{1-\delta} \geqslant \frac{\sum_{i=1}^{n} \pi\left(r_{i}\right)}{\sum_{i=1}^{n}\left[\pi\left(r_{1}\right)-\pi\left(r_{i}\right)\right]}$. 
On the other hand, (4) and the fact that $\left(\sum r_{i}\right) / n-\left(\sum \hat{r}_{i}\right) / n=\delta\left[\left(\sum r_{i}\right) / n-r_{1}\right]$ together imply that

$$
\frac{1-\delta^{T}}{\delta^{T+1}}=\frac{\left(\sum r_{i}\right) / n-r_{1}}{Y-\left(\sum r_{i}\right) / n},
$$

Combining this equality with (5), the required condition becomes

$$
r_{n}-\left(\sum r_{i}\right) / n>Y-\left(\sum r_{i}\right) / n,
$$

which is clearly the case when $Y<r_{n}$.

So the question of average agent payoffs cannot be resolved by simply studying the standard-offer equilibrium. Equilibria with delay may yield higher agent payoffs, and what is more, we've seen that as all agents approach infinite patience, equilibrium delay can grow without bound. Does this mean that (normalized) agent payoffs approach $r_{n}$ ?

Clearly, the answer to the question raised in the previous paragraph must turn on how quickly the delay approaches infinity as the discount factor tends to one. This is because an increase in the discount factor forces agent payoffs to depend more sensitively on the far future (when the agents will finally be contracted by the principal). At the same time, this far future is growing ever more distant, by Proposition 3. In addition, there are other concerns. Proposition 3 only discussed the initial delay, but there may be equilibria involving various sets of delays, interspersed with accepted offers. Proposition 4 tells us, however, that all these effects can be brought together.

Proposition 4. There exists $\eta>0$ such that the supremum of average agent payoffs over all equilibria and all discount factors cannot exceed $r_{n}-\eta$.

This proposition establishes conclusively that, despite the fact that delays can become arbitrarily large, normalized average payoffs for the agent stay below and bounded away from the "fully free" payoff, which is $r_{n}$. The principal's overtures can be resisted to some extent, but not fully.

The proof of Proposition 4 employs a recursive argument. Along any equilibrium, whenever there are $m$ free agents left at any stage, Proposition 3 provides a bound on the maximal delay permissible at that stage. Moreover, when some agents are contracted after the delay, there are bounds on what they can receive from the principal. The proof proceeds by taking a simple average of all these (delayed) payoffs, and then completes the argument by noting that while the delay $T$ may be going to infinity as $\delta$ goes to one, Proposition 3 pins down the combined term $\delta^{T}$. This permits us to conclude that the present value of the bounds are uniform in the discount factor. ${ }^{11}$

We end this section with some comments on the robustness of our results.

As before, these results generalize to the use of nonlinear profit functions that are increasing in the number of bonded agents. The principal's worst and best equilibrium payoff would

\footnotetext{
11 This final step appears to rely on the fact that the agents and the principal have the same discount factor. It remains to be seen how this result would look for the case of different discount factors.
} 
still be his payoff from the standard offer and from the low offer respectively (though these payoffs would naturally be different), and therefore the main results would be unaffected.

One may also speculate if our results are robust to contracts that last only for a finite duration. [This would happen, for instance, if the principal cannot make offers that are binding beyond the current period.] To be sure, new equilibria might then appear, but the bounds that we have identified remain unchanged. The reasoning is straightforward, so we only provide a brief outline. The first step is to note that the standard offer is immune to agents leaving a contract, even if they can do so (once again, an argument based on iterated deletion of dominated strategies will apply). It follows that the standard-offer equilibrium continues to remain an equilibrium. Given this, our main source of agent punishment remains unaffected: it is possible to deter a group of agents who would leave the principal by a standard-offer equilibrium with the lowest offer going to the group member who had the highest offer to start with. In particular, the low-offer equilibrium is unaffected by the consideration of contracts with finite lifetimes. So is the fact that this is the best equilibrium available to the principal. The main propositions now go through just as before.

\section{Conclusion}

A principal interacts with several agents by offering them contracts. We assume that all contracts are bilateral, and that the principal's payoff from a package of contracts is the sum of payoffs from the individual contracts. Our specification of a contract is reduced-form: it stipulates a net payoff pair to the principal and agent. The crucial assumption of this paper is that the outside-option payoffs of the agents depend positively on how many uncontracted or "free" agents there are. Indeed, such positive externalities imply that the agents are better off not having the principal around in the first place.

To be sure, the principal can still make substantial profits by relying on coordination failure among the agents. However, we explicitly rule out problems of coordination. This paper shows, nevertheless, that in a dynamic framework, agents must "eventually" succumb to the contracts offered by the principal—and often at inferior terms.

This result is obtained in two versions of the model. In the first model, the principal cannot return to an agent who has earlier refused him, while in the second, the principal can return to an agent as often as he wishes. In both models, time plays an explicit role in the description of the equilibria of interest. If the principal can commit, there is a unique perfect equilibrium, in which contract provision is divided into two phases. In the first phase, simultaneous and relatively attractive offers are made to a number of agents, though the offers must generally vary among the recipients. In the second, "exploitative" phase, offers are made (and accepted) sequentially, and their values are "discontinuously" lower than those of the first phase. In fact, the payoffs received by agents in this phase are close to the very lowest of all the outside options. So, in this model, a community of agents is invaded in two phases: some agents are bought, the rest are "exploited", in the sense that at the time of their contract, their outside option is strictly higher than what they receive.

In the second version of the model, there is a multiplicity of equilibria. In some of these outcomes every agent succumbs to the principal immediately, though with different payoff consequences depending on the equilibrium in question. But there are other equilibria in 
which the agents have the power to force delay. Nevertheless, we show that they cannot hold the principal off forever; in every perfect equilibrium, agents must succumb in finite time. Indeed, even if the delay becomes unboundedly large as the discount factor approaches one, our final result shows that the payoff of the agents must stay below and bounded away from the fully free reservation payoff.

These results have implications for the way we think about markets and equilibrium in the context of externalities. They also allow us to define a notion of "exploitation" which may be useful, at least in certain development contexts. Development economists have long noted that while informal contractual arrangements may be extremely unequal, it is hard to think of them as being exploitative as long as outside opportunities are respected. In this paper, outside opportunities are respected but they are endogenous, and are affected by the principal's past dealings. It is in this sense-in the deliberate altering of outside options - that the principal's actions may be viewed as "exploitative". ${ }^{12}$

\section{Proofs}

\subsection{The single-offer model}

In this section, we look at the case in which offers to a particular agent cannot be made more than once.

Proof of Proposition 6. First we choose $\hat{\delta}$. Pick any $\varepsilon \in\left(0, \min _{i}\left\{r_{i+1}-r_{i}\right\}\right)$. Now choose $\hat{\delta} \in(0,1)$ such that for all $\delta \in(\hat{\delta}, 1)$,

$$
\left(1-\delta^{n}\right) r_{n}+\delta^{n} r_{i} \leqslant r_{i}+\varepsilon
$$

and

$$
\left(\delta^{n-k}+\cdots+\delta^{n}\right) \pi\left(r_{i}+\varepsilon\right)>\sum_{j=i}^{i+k} \pi\left(r_{j}\right) \text { for any } k \in\{1, \ldots, n-1\}
$$

for all $i=1, \ldots, n-1$. Because $r_{i+1}>r_{i}$ and $\pi(r)$ is strictly decreasing, it is always possible to choose $\hat{\delta}$ such that both these requirements are met.

Throughout this proof, we assume $\delta \geqslant \hat{\delta}$.

By a minigame we will refer to a collection $(m, \mathbf{z})$, where $1 \leqslant m \leqslant n$, and $\mathbf{z}=\left(z_{1}, \ldots, z_{m}\right)$ is a vector of reservation payoffs for the $m$ agents arranged in increasing order. Throughout, a minigame will have no more than $n$ agents, and $\mathbf{z}$ will always be drawn from a "connected string" of the $r_{i}$ 's in the original game. To illustrate, $\left(z_{1}, \ldots, z_{m}\right)$ could be $\left(r_{1}, \ldots, r_{m}\right)$, or $\left(r_{3}, \ldots, r_{m+2}\right)$.

Imagine that one agent accepts an offer at this minigame. This induces a fresh minigame $(m-1, \mathbf{z})$, where it is understood that $\mathbf{z}$ now refers to the vector $\left(z_{1}, \ldots, z_{m-1}\right)$. We then say that $(m, \mathbf{z})$ positively induces $(m-1, \mathbf{z})$.

\footnotetext{
${ }^{12}$ The work of Basu in [3] is related to this view, though the channels he studies are different.
} 
Now imagine that a single agent refuses an offer (and only one offer is made) at $(m, \mathbf{z})$. This induces a fresh minigame $\left(m-1, \mathbf{z}^{\prime}\right)=(m-1, \psi(\mathbf{z}))$, where it is understood that $\mathbf{z}^{\prime}=\psi(\mathbf{z})$ now refers to the vector $\left(z_{2}, \ldots, z_{m}\right)$. We then say that $(m, \mathbf{z})$ negatively induces $(m-1, \psi(\mathbf{z}))$.

The proof proceeds by induction on the number of agents in any minigame. We shall suppose that the properties listed below hold for all stages of the form $(m, \mathbf{z})$, where $2 \leqslant m \leqslant M$ and $\mathbf{z}$ is some arbitrary vector of reservation payoffs (but a substring of $\mathbf{r}$ as discussed). We shall then establish these properties for all stages of the form $(M+1, \mathbf{z})$.

Induction hypothesis. [A] For all stages $(m, \mathbf{z})$ with $1 \leqslant m \leqslant M$, there is a unique equilibrium. Either no acceptable offers are made, or all agents accept offers in the equilibrium, and the principal makes non-negative profits.

Before proceeding further, some definitions. Let $P(m, \mathbf{z})$ denote the principal's profit at any such minigame $(m, \mathbf{z})$. For $m \geqslant 2$ but no bigger than $M+1$, say that a minigame $(m, \mathbf{z})$ is pivotal if it negatively induces the minigame $\left(m-1, \mathbf{z}^{\prime}\right)$, and the principal makes no acceptable offers in that minigame. Otherwise $(m, \mathbf{z})$ is not pivotal. (Note that a definition of pivotality is included for stages of the form $(M+1, \mathbf{z})$.)

We now continue with the description of the induction hypothesis.

[B] If for $2 \leqslant m \leqslant M,(m, \mathbf{z})$ is a pivotal minigame, then look at the minimal $k \leqslant m$ such that $(k, \mathbf{z})$ is pivotal. ${ }^{13}$ Then either the principal makes no acceptable offers at that minigame, or - if the principal's payoff under the description that follows is non-negativethe principal makes simultaneous offers to $m-k+1$ agents, and begins the play of the minigame $(k-1, \mathbf{z})$ at the same time. The simultaneous offers to the $m-k+1$ agentscall them agents $k, \ldots, m$-satisfy the property that agent $j$ (in this group) receives the offer $z_{j}$.

[C] If for $2 \leqslant m \leqslant M,(m, \mathbf{z})$ is not a pivotal minigame, the principal makes a single offer to each agent, one period at a time, which are all accepted. Each agent's payoff lies in the range $\left[z_{1}, z_{1}+\varepsilon\right]$, where $\varepsilon$ was chosen at the start of this proof (see (6) and (7)).

The following lemmas will be needed.

Lemma 1. Suppose that $2 \leqslant m \leqslant M$, and that [A] of the induction hypothesis holds. (1) If the principal makes no acceptable offers during the minigame $(m, \mathbf{z})$, then at its negatively induced minigame ( $m-1, \psi(\mathbf{z}))$, the principal makes no acceptable offers as well. (2) If the principal makes equilibrium offers at the minigame $(m, \mathbf{z})$, then at its positively induced minigame $(m-1, \mathbf{z})$, he does so as well.

Proof. (1) Suppose not, so that the principal makes acceptable equilibrium offers at the minigame $(m, \psi(\mathbf{z}))$. Consider the strategy followed by the principal at this minigame, and follow exactly this strategy for the minigame $(m, \mathbf{z})$, ignoring one of the agents completely. It must be the case that all $m-1$ agents behave exactly as they did in the minigame $(m-1, \psi(\mathbf{z}))$. By $[\mathrm{A}]$ and our convention that offers are made when profits are non-negative, all agents are thereby contracted. Finally, offer the ignored agent $z_{1}$; he will accept. The

\footnotetext{
${ }^{13}$ Recall that $\mathbf{z}$ is now to be interpreted as the old vector of reservation payoffs up to the first $k$ terms.
} 
principal's total return from this feasible strategy is therefore $P\left(m-1, \mathbf{z}^{\prime}\right)+\delta^{T} \pi\left(z_{1}\right)>0$, where $T$ is the time it takes to contract the $m-1$ agents. [This is because $P\left(m-1, \mathbf{z}^{\prime}\right) \geqslant 0$ and so $\pi\left(z_{1}\right)>0$.] But $P(m, \mathbf{z}) \geqslant P\left(m-1, \mathbf{z}^{\prime}\right)+\delta^{T} \pi\left(z_{1}\right)$, which implies that $P(m, \mathbf{z})>0$, a contradiction.

(2) Consider the strategy followed by the principal at $(m, \mathbf{z})$, and follow exactly this strategy for the minigame $(m-1, \mathbf{z})$, simply deleting the highest offer made. It is optimal for the $m-1$ agents to stick to exactly the same strategy that they used before. Therefore this strategy yields the principal a possible payoff of $P(m, \mathbf{z})-\delta^{T} \pi\left(z^{*}\right)$, where $z^{*}$ was the highest offer made and $T$ the date at which it was made. We can see that

$$
P(m-1, \mathbf{z}) \geqslant P(m, \mathbf{z})-\delta^{T} \pi\left(z^{*}\right) .
$$

Now the right-hand side of $(8)$ is clearly non-negative if $\pi\left(z^{*}\right) \leqslant 0$. But it is also nonnegative when $\pi\left(z^{*}\right) \geqslant 0$, because the amount $\delta^{T} \pi\left(z^{*}\right)$ is already included in $P(m, \mathbf{z})$, and the remainder, consisting of lower offers to the agents, must yield non-negative payoff to the principal.

Lemma 2. Assume [A], and let $2 \leqslant m \leqslant M$. Then if $(m+1, \mathbf{z})$ is not pivotal, its positively induced minigame $(m, \mathbf{z})$ cannot be pivotal either.

Proof. If $(m+1, \mathbf{z})$ is not pivotal, this means that its negatively induced minigame $(m, \psi(\mathbf{z}))$ has the principal making equilibrium offers. By (2) of Lemma 1, the positively induced minigame from $(m, \psi(\mathbf{z}))$, which is $(m-1, \psi(\mathbf{z}))$, has the principal making equilibrium offers as well. But $(m-1, \psi(\mathbf{z}))$ is also the negatively induced minigame of $(m, \mathbf{z})$. This means that $(m, \mathbf{z})$ cannot be pivotal.

Proof of the induction step. Our goal is to establish $[\mathrm{A}]-[\mathrm{C}]$ for minigames of the form $(M+1, \mathbf{z})$. Suppose, first, that $(M+1, \mathbf{z})$ is a pivotal minigame. We claim that if any acceptable offers are made at all, then at least one agent must be offered $z_{M+1}$ or more. To see this, let $s$ agents be made offers at the first date. If no agent is offered $z_{M+1}$ or more, their equilibrium strategy is to reject. For this will induce the minigame $\left(M+1-s, \psi^{s}(\mathbf{z})\right)$, where $\psi^{s}$ is just the $s$-fold composition of $\psi$. Remember that $(M+1, \mathbf{z})$ is pivotal, so that in $(M, \psi(\mathbf{z}))$ no acceptable offers can be made. By applying Lemma 1, part (1), repeatedly, we must conclude that no acceptable offers can be made at the minigame $\left(M+1-s, \psi^{s}(\mathbf{z})\right)$. Because two or more offers cannot be made, this means that all agents enjoy $z_{M+1}$, their highest possible payoff. But now we have shown that no acceptable offers are made at all, which is a contradiction.

So the claim is true: if any acceptable offers are made, then at least one agent must be offered $z_{M+1}$ or more. It is obvious that an offer of exactly $z_{M+1}$ need be made in equilibrium. Suppose this offer is indeed made. Notice that there is positive profit to be made from the remaining $M$ agents. ${ }^{14}$ By discounting, the minigame $(M, \mathbf{z})$ must be started as soon as possible (now!).

\footnotetext{
${ }^{14}$ If $\pi\left(z_{M+1}\right) \geqslant 0$ this is certainly true. But if $\pi\left(z_{M+1}\right)<0$ this is also true, because no remaining agent will be offered more than $z_{M}$, which is strictly less than $z_{M+1}$.
} 
Now look at this minigame $(M, \mathbf{z})$, to which the induction hypothesis applies. If it is not pivotal, then by Lemma 2, no positively induced minigame of it can be pivotal as well. In this case the minimal pivotal $k$ such that $(k, \mathbf{z})$ is pivotal is exactly $M+1$, and [A] and [B] have been established. ${ }^{15}$

If, on the other hand, the minigame $(M, \mathbf{z})$ is pivotal, then, too, [A] and [B] have been established, because we have shown that the principal must immediately start this minigame along with the offer of $z_{M+1}$ to the first agent.

Now, we establish $[\mathrm{A}]$ and $[\mathrm{C}]$ in the case where $(M+1, \mathbf{z})$ is not a pivotal minigame. First suppose that the principal makes a single offer, which is refused. By non-pivotality, all agents will be contracted in the negatively induced minigame $(M, \psi(\mathbf{z}))$. The payoff to the single agent who refused, therefore, cannot exceed

$$
\left(1-\delta^{n}\right) z_{n}+\delta^{n} z_{1} \leqslant\left(1-\delta^{n}\right) r_{n}+\delta^{n} z_{1},
$$

where $n$, it will be recalled, is the grand total of all agents. Using (6) and remembering that $z_{1}=r_{i}$ for some $i$, we must conclude that our single agent must accept some offer not exceeding $z_{1}+\varepsilon$. So the principal, if he so wishes, can generate a payoff of at least $\pi\left(z_{1}+\varepsilon\right)+\delta P(M, \mathbf{z})$. But by Lemma 2 , if $(M+1, \mathbf{z})$ is not pivotal, its positively induced minigame $(M, \mathbf{z})$ cannot be pivotal either. By the induction hypothesis applied to this minigame (see part [C]), we may conclude that the principal makes a single accepted offer to each agent, one period at a time, and that each agent's offer lies in the range $\left[z_{1}, z_{1}+\varepsilon\right]$. So the principal's payoff at the minigame $(M+1, \mathbf{z})$ is bounded below by

$$
\pi\left(z_{1}+\varepsilon\right)\left(1+\delta+\cdots+\delta^{M+1}\right) .
$$

What are the principal's other alternatives? He could attempt, now, to make an offer to some set of agents instead, say of size $s>1$. If $s^{\prime} \leqslant s$ of these agents refuse the offers, they would be assured of a long-term payoff of at least $z_{s}^{\prime}$. Using the agent coordination criterion, it follows that to get these $s$ agents to accept, the principal would therefore have to offer at least the vector $\left(z_{1}, \ldots, z_{s}\right)$. The minigame that remains is just $(M+1-s, \mathbf{z})$, which continues to be non-pivotal by Lemma 2. By applying the induction hypothesis, the additional payoff to the principal here is

$$
\pi\left(z_{1}+\varepsilon\right)\left(1+\delta+\cdots+\delta^{M+1-s}\right) .
$$

so the total payoff under this alternative is bounded above by

$$
\sum_{j=1}^{s} \pi\left(z_{j}\right)+\pi\left(z_{1}+\varepsilon\right)\left(1+\delta+\cdots+\delta^{M+1-s}\right) .
$$

Compare (9) and (10). The former is larger if

$$
\pi\left(z_{1}+\varepsilon\right)\left(\delta^{M+2-s}+\cdots+\delta^{M+1}\right)>\sum_{j=1}^{s} \pi\left(z_{j}\right) .
$$

\footnotetext{
15 Note: The uniqueness makes use of our convention that indifference in all cases results in positive action, whether in the making or in the acceptance of offers.
} 
Recalling that $\mathbf{z}$ is always drawn as a "substring" of $\mathbf{r}$, and invoking (7) (by setting $k=s-1$ and noting that $n \geqslant M+1$ ), we see that this inequality must be true. So the alternative is worse.

Finally, there is an obvious loss to the principal in making offers to agents and deliberately have them refuse. So all options are exhausted, and $[\mathrm{A}]$ and $[\mathrm{C}]$ of the induction step are established.

Starting point: All that is left to do is to establish the validity of the induction step when $m=2$, and for any vector $\left(z_{1}, z_{2}\right)$ (substrings of $\mathbf{r}$, of course). Using (6) and (7) once again, this is a matter of simple computation.

The last step needed to complete the proof of the proposition consists in proving that $m(\delta)$ is non-decreasing in $\delta$. This is equivalent to proving the following lemma.

Lemma 3. For any minigame $(m, \mathbf{z})$, if $(m, \mathbf{z})$ is non-pivotal under $\delta$ then it is non-pivotal under $\delta^{\prime}>\delta$.

Proof. Let us use an inductive argument. It is straightforward to check that if $(2, \mathbf{z})$ is non-pivotal under $\delta$, then it is non-pivotal under any $\delta^{\prime}>\delta$.

Assuming the lemma is true for all stages of the form $(m, \mathbf{z})$ for $2 \leqslant m \leqslant M$ and all $\mathbf{z}$, we need to prove that this property holds for all stages $(M+1, \mathbf{z})$. Suppose not, so that $(M+1, \mathbf{z})$ is non-pivotal under $\delta$ but pivotal under $\delta^{\prime}>\delta$. That is $P_{\delta}(M, \psi(\mathbf{z})) \geqslant 0 P_{\delta^{\prime}}(M, \psi(\mathbf{z}))<$ 0 , where the subscript indicates the dependence on the discount rate. Denote as $k^{*}(\delta)$ the minimal $k \leqslant M$ such that $(k, \psi(\mathbf{z}))$ is pivotal given $\delta$. Since Lemma 3 is true for all $2 \leqslant m \leqslant M$ and $\mathbf{z}$, it must be that $k^{*}(\delta)<k^{*}\left(\delta^{\prime}\right)$. Using this and using the first part of the proposition to compute the principal profit it is clear that $P_{\delta^{\prime}}(M, \psi(\mathbf{z})) \geqslant P_{\delta}(M, \psi(\mathbf{z}))$, which is a contradiction. ${ }^{16}$

Now the proof of the proposition is complete.

\subsection{The model with multiple offers}

We now turn to the case in which the principal can repeatedly approach agents.

Proof of Proposition 2. It is obvious that in no equilibrium can the principal's payoff fall below the bound described in (1). For suppose, on the contrary, that this were indeed the case at some equilibrium; then the principal could make an offer $\mathbf{c}$ with each component $c_{i}$ exceeding the corresponding component $r_{i}$ of the standard offer by a $\varepsilon_{i}>0$. Clearly agent $n$ cannot resist such an offer (for in no equilibrium can she get strictly more than $r_{n}$ ). But then nor can agent $n-1$ resist her component of the offer, and by an iterative argument all agents must accept the offer $\mathbf{c}$. If the $\varepsilon_{i}$ 's are small enough, this leads to a contradiction.

So it must be that the principal can guarantee at least $\sum_{i=1}^{n} \pi\left(r_{i}\right)$ in any equilibrium. It remains to show that there are equilibria in which the payoff of $\sum_{i=1}^{n} \pi\left(r_{i}\right)$ is attained.

\footnotetext{
${ }^{16}$ Note that the first part of the proposition applies since, if $\varepsilon$ satisfies (6) and (7) under $\delta$ then it satisfies them under $\delta^{\prime}$ too.
} 
Notice that an agent must reject any offer of strictly less than $r_{1}$. We can, without loss of generality, treat a no-offer as equivalent to an offer of less than $r_{1}$. In what follows, this will ease notation: we can think, when convenient, of the principal as always making an offer to every free agent in every subgame.

Our proof proceeds by describing three kinds of phases.

Normal phase: If there are $m$ free agents, the principal makes them the standard offer. All agents must accept.

The following deviations from the normal phase are possible:

[A] Some agents reject. In that case, "reverse-name" the rejectors so that the rejector with the highest label is now agent 1 , the rejecting agent with the next-highest label is now 2 , and so on. Restart with normal phase with these free agents.

[B] The principal deviates with a different offer $\mathbf{c}$, where we arrange the components so that $c_{1} \leqslant c_{2} \leqslant \cdots \leqslant c_{n}$. Rename the agents so that component $i$ is given to agent $i$, and proceed to the evaluation phase; see below.

c-Evaluation phase: Given an offer of $\mathbf{c}$ made to $n$ agents, this phase proceeds as follows. Define $K$ to be the largest integer $k \in\{1, \ldots, n\}$, such that $c_{i}<(1-\delta) r_{k}+\delta r_{i}$ for all $1 \leqslant i \leqslant k$, and if this condition cannot be satisfied for any $k \geqslant 1$, set $K=0$. All the agents between 1 and $K$ must reject their offers. If, in addition,

$$
\sum_{i=K+1}^{n} \pi\left(c_{i}\right) \leqslant \sum_{i=K+1}^{n} \pi\left(r_{i}\right),
$$

the agents with indices larger than $K$ must accept the offer. Proceed to the normal phase with the $K$ agents, if any. If, on the other hand,

$$
\sum_{i=K+1}^{n} \pi\left(c_{i}\right)>\sum_{i=K+1}^{n} \pi\left(r_{i}\right)
$$

then define $L$ as the smallest integer $\ell \in\{1, \ldots, n-1\}$ such that $c_{i}>r_{i}$ for all $i>\ell$, and if this condition cannot be satisfied for any $\ell \geqslant 1$, set $L=n .{ }^{17}$ All agents 1 to $L$ must reject the offer while agents with indices larger than $L$ must accept the offer. Let $\widetilde{\mathbf{c}} \equiv\left\{c_{1}, \ldots, c_{L}\right\}$. Now proceed to the $\widetilde{\mathbf{c}}$-punishment phase; see below.

The following deviations from the evaluation phase are possible:

[A] Some agents accept when asked to reject; and/or some reject when asked to accept. If there are $m \geqslant 1$ rejectors following these deviations, rename agents from 1 to $m$ respecting the original order of their names; go to the normal phase.

$\widetilde{\mathbf{c}}$-Punishment phase: Recall that an offer of $\widetilde{\mathbf{c}} \in \mathbb{R}^{L}$ is on the table and that agents are named as in the evaluation phase. Define $T \geqslant 0$ to be the largest integer such that

$$
\delta^{T+1}\left[\sum_{i=1}^{K} \pi\left(r_{i}\right)+\sum_{i=K+1}^{L} \pi\left(c_{i}\right)\right] \leqslant \sum_{i=1}^{L} \pi\left(r_{i}\right),
$$

\footnotetext{
${ }^{17}$ Note that $L>K$ for otherwise (12) is contradicted.
} 
while at the same time,

$$
\delta^{T}\left[\sum_{i=1}^{K} \pi\left(r_{i}\right)+\sum_{i=K+1}^{L} \pi\left(c_{i}\right)\right] \geqslant \sum_{i=1}^{L} \pi\left(r_{i}\right) .
$$

The principal must now wait for $T$ periods, making no offer at all (or offer of strictly less than $\left.r_{1}\right)$. Following the $T$ periods he makes the offer $\left(r_{1}, \ldots, r_{K}, c_{K+1}, \ldots, c_{L}\right)$, which is to be unanimously accepted.

The following deviations from the $\widetilde{\mathbf{c}}$-punishment phase are possible:

[A] If any agents reject the final offer, "reverse-name" the rejectors so that the rejector with the highest label is now agent 1 , the rejecting agent with the next-highest label is now 2 , and so on. Proceed to the normal phase with these rejecting agents as the free agents.

[B] If the principal deviates in any way, by making an offer of $\mathbf{c}^{\prime} \in \mathbb{R}^{L}$, start a $\mathbf{c}^{\prime}$-evaluation phase.

We now prove that this description constitutes an equilibrium which is, moreover, immune to coordinated deviations by the agents.

Begin with deviations in the normal phase. Suppose that a group of $s \geqslant 1$ agents reject their components of the standard offer; let $j$ be the agent with the highest rank in that group. Given the prescription of play, he will be offered (and will accept) $r_{1}$ next period. His overall return is, therefore,

$$
(1-\delta) r_{s}+\delta r_{1}
$$

where is the number of rejectors. Because $s \leqslant j$, we see that $r_{s} \leqslant r_{s}$. Of course, $r_{1} \leqslant r_{s}$. Consequently, the agent's payoff is no higher than $r_{s}$, which is what he is offered to start with. Therefore no agent deviation, coordinated or otherwise, can improve the well-being of every deviating agent.

Now suppose that the principal deviates with offer c. The prescription then takes us to the evaluation phase. If (11) applies, then agents with indices larger than $K$ accept, and the remaining agents accept in the normal phase one period after that. Consequently, the principal's payoff is given by

$$
\sum_{i=K+1}^{n} \pi\left(c_{i}\right)+\delta \sum_{i=1}^{K} \pi\left(r_{i}\right) \leqslant \sum_{i=K+1}^{n} \pi\left(c_{i}\right)+\sum_{i=1}^{K} \pi\left(r_{i}\right) \leqslant \sum_{i=1}^{n} \pi\left(r_{i}\right),
$$

where the first inequality follows from (A.1) and the fact that $\pi$ is decreasing (so that $\left.\sum_{i=1}^{K} \pi\left(r_{i}\right) \geqslant 0\right)$, and the second inequality is a direct consequence of (11). So this deviation is not profitable.

If (12) applies in the evaluation phase, then only agents with indices larger than $L$, if any, accept. The remaining agents must reject and we proceed thereafter with these agents to a further wait of $T$ periods (where $T$ is defined by (13) and (14)), followed by an offer of $\left(r_{1}, \ldots, r_{K}, c_{K+1}, \ldots, c_{L}\right)$, which they accept. Therefore, the principal's payoff is 
given by

$$
\delta^{T+1}\left[\sum_{i=1}^{K} \pi\left(r_{i}\right)+\sum_{i=K+1}^{L} \pi\left(c_{i}\right)\right]+\sum_{i=L+1}^{n} \pi\left(c_{i}\right) \leqslant \sum_{i=1}^{N} \pi\left(r_{i}\right),
$$

by (13) and the definition of $L$. Once again, the deviation is not profitable. This completes our verification in the normal phase.

Turn now to the evaluation phase. Suppose, first, that some agent accepts an offer when he has been asked to reject. Let $i$ and $j$ be the deviating agent with the lowest and largest index respectively. First, consider the case in which $i \leqslant K$. His return from the deviation is $c_{i}$. In contrast, if all deviators were to stick to the prescribed path, agent $i$ would receive $a t$ least

$$
(1-\delta) r_{K}+\delta r_{i}
$$

(it would be even more if (12) were to hold). By the definition of $K, c_{i}<(1-\delta) r_{K}+\delta r_{i}$. It follows that there is always some agent in the set of $K$ agents who would not be better off by participating in any deviation, coordinated or unilateral.

Next, consider the case in which (12) holds and an agent $i$ with $K<i \leqslant L$ accepts the offer. Along the prescribed play, agent $j$ 's payoff is

$$
\left(1-\delta^{T+1}\right) r_{L}+\delta^{T} c_{j}
$$

which is at least as much as $c_{j}$, his return from the deviation. Indeed, otherwise, if $r_{L}<c_{j}$, then $r_{L}<c_{L}$ which contradicts the definition of $L$.

Now, assume that a set $S$ of $m$ agents reject offers when they were supposed to accept them. Pick the deviating agent with the largest index, say $j$. Note that, if $j>L$, this is clearly not a profitable deviation since $c_{j}>r_{j}$. So we need only consider deviations with $j \leqslant L$, and therefore situations in which (11) applies. Let us index the agents who rejects the offer from 1 to $m$ respecting the original order of their names, and let $\mu(i) \in\{1, \ldots, m\}$ be the new index for an agent with original index $i$. By deviating, agent $i \in S$ receives $(1-\delta) r_{m}+\delta r_{\mu(i)}$. Note that for at least one $i \in S$, it must be that

$$
(1-\delta) r_{m}+\delta r_{\mu(i)} \geqslant c_{i}
$$

otherwise this would contradict the fact that $i>K$. It follows that there is always some agent in the set of $S$ agents who would not be better off by participating in this deviation.

Finally, consider a c-punishment phase. Suppose that a group of agents rejects the final offer. Let $k$ be the highest index in that group. Recalling the subsequent prescription, this individual receives, by rejecting, no more than $(1-\delta) r_{k}+\delta r_{1}$ (and may receive less if some agent below $k$ does accept). If $k \leqslant K$, this agent receives $r_{k}$ in the event of no deviation, which cannot be lower. If $k>K$, he is supposed to receive $c_{k}$ in the event that no one deviates. We claim that

$$
c_{k} \geqslant(1-\delta) r_{k}+\delta r_{1} .
$$

Suppose not, then $c_{k}<(1-\delta) r_{k}+\delta r_{1} \leqslant(1-\delta) r_{k}+\delta r_{i}$ for all $i \leqslant k$. This contradicts the fact that $k>K$, so the claim is proved. We have therefore shown that there is always some participating agent who is not made better off by a deviation, coordinated or not. 
Now suppose the principal deviates at any stage of the punishment phase with $M$ periods of waiting left, where $0 \leqslant M \leqslant T$. Suppose she offers $\mathbf{c}^{\prime}$. Now the evaluation phase is invoked. Denote by $K^{\prime}$ the corresponding construction of $K$ for $\mathbf{c}^{\prime}$. If (11) holds (for $\mathbf{c}^{\prime}$ ), then the principal's return is

$$
\begin{aligned}
\sum_{i=K^{\prime}+1}^{L} \pi\left(c_{i}^{\prime}\right)+\delta \sum_{i=1}^{K^{\prime}} \pi\left(r_{i}\right) & \leqslant \sum_{i=K^{\prime}+1}^{L} \pi\left(c_{i}^{\prime}\right)+\sum_{i=1}^{K^{\prime}} \pi\left(r_{i}\right) \\
& \leqslant \sum_{i=1}^{L} \pi\left(r_{i}\right) \\
& \leqslant \delta^{T}\left[\sum_{i=1}^{K} \pi\left(r_{i}\right)+\sum_{i=K+1}^{L} \pi\left(c_{i}\right)\right] \\
& \leqslant \delta^{M}\left[\sum_{i=1}^{K} \pi\left(r_{i}\right)+\sum_{i=K+1}^{L} \pi\left(c_{i}\right)\right],
\end{aligned}
$$

where the first inequality follows from (A.1), as before, the second from (11), the third from (14), and the last from the fact that $M \leqslant T .{ }^{18}$ Because the final expression is the payoff from not deviating, we see there is no profitable deviation in this case.

Alternatively, (12) holds for $\mathbf{c}^{\prime}$. Denote by $L^{\prime}$ the corresponding construction of $L$ for $\mathbf{c}^{\prime}$. Then agents $L^{\prime}+1$ to $L$ accept the offer while all other offers must be rejected. Let $T^{\prime}$ be the further wait time as a new punishment phase starts up. Applying the prescription, the principal's payoff is

$$
\begin{aligned}
\sum_{i=L^{\prime}+1}^{L} \pi\left(c_{i}^{\prime}\right)+\delta^{T^{\prime}+1}\left[\sum_{i=1}^{K^{\prime}} \pi\left(r_{i}\right)+\sum_{i=K^{\prime}+1}^{L^{\prime}} \pi\left(c_{i}^{\prime}\right)\right] & \leqslant \sum_{i=1}^{L} \pi\left(r_{i}\right) \\
& \leqslant \delta^{T}\left[\sum_{i=1}^{K} \pi\left(r_{i}\right)+\sum_{i=K+1}^{L} \pi\left(c_{i}\right)\right] \\
& \leqslant \delta^{M}\left[\sum_{i=1}^{K} \pi\left(r_{i}\right)+\sum_{i=K+1}^{L} \pi\left(c_{i}\right)\right]
\end{aligned}
$$

where the first inequality follows from (13) applied to $T^{\prime}$ and the definition of $L^{\prime}$, and the remaining inequalities follow exactly the same way as in (15). Here, too, no deviation is profitable.

Thus far we have established the first part of the proposition; see (1). Now we turn to (2).

First, we show that an equilibrium exists in which the principal's payoff is $\bar{P}=\sum_{i=1}^{n} \pi\left(\hat{r}_{i}\right)$. It suffices to show that an equilibrium exists in which the low offer is made and accepted. It is supported by two phases, the low-offer phase and the standard-offer phase, and the

\footnotetext{
18 To perform this final step, we also need to reassure ourselves that the last expression is positive, but that is automatically guaranteed by the presence of the third expression in (15), and (A.1).
} 
following prescriptions are in force:

(1) Begin with the low-offer phase. The low offer is made to all agents and should be accepted. If the principal conforms but there are any rejections, proceed to the standardoffer phase with a suitable renaming of agents (see below). If the principal makes a different offer, move to the standard-offer phase with no renaming of the free agents.

(2) In the low-offer phase, if $m$ agents receive an offer $\mathbf{c}$, where we arrange the components so that $c_{1} \leqslant c_{2} \leqslant \cdots \leqslant c_{m}$, then let $j \in\{0, \ldots, m\}$ be the lowest index such that $c_{i}>\hat{r}_{i}$ for all $i>j$ or 0 if no such index exists. All agents with index $i>j$, if any, accept the offer; all others reject. Move to the standard-offer phase with suitable renaming (see below) if some agent who is meant to accept rejects the offer.

(3) In any subgame where the standard-offer phase is called for, and renaming is required, identify the rejectors who were meant to accept their offers. "Reverse-name" the rejectors so that the rejector with the highest label is now agent 1 , the rejecting agent with the next-highest label is now 2, and so on. If there are any other free agents, give them higher labels. If no renaming is required, ignore above instructions. Now play a standard-offer equilibrium with all these agents.

To examine whether this constitutes an equilibrium, consider agent strategies first. It suffices to consider only the low-offer phase. All agents have been made an offer. If $k$ agents jointly reject, the subsequent payoff to the agent with the highest offer is $r_{k}$ today (because there are exactly $k$ free agents after the rejection), followed by $r_{1}$ in the next period (by virtue of item [3]). This means that such an agent would accept any offer that exceeds $\hat{r}_{k}=(1-\delta) r_{k}+\delta r_{1}$. Hence, by iterated deletion of dominated strategies the agents would all accept the offers $\left(\hat{r}_{1}, \ldots, \hat{r}_{n}\right)$.

As for the principal, consider again the low-offer phase. Offering more than $\left(\hat{r}_{1}, \ldots, \hat{r}_{n}\right)$ in any component is not a profitable deviation. Making acceptable offers to less than the full set of free agents just precipitates the standard offer equilibrium thereafter, which certainly makes him worse off. Finally, making unacceptable offers to a set of $k$ agents again triggers the standard-offer phase and a continuation profit of $\underline{P}(k)$ which is the lowest possible continuation profit on $k$ agents. Clearly, the principal does not have incentive to deviate from his prescribed actions.

Finally, to see that this is the highest payoff that the principal could ever receive, it suffices to recall that $r_{1}$ is the worst possible continuation utility an agent can ever receive. Hence, to sign on any agent when $n$ agents are free, the principal has to make at least one offer of $\hat{r}_{n}$. Given this, whether in this period or later, the principal cannot offer anything less than $\hat{r}_{n-1}$ in order to sign up another agent. By repeating the argument for all remaining agents, and recalling that delays are costly, we see that $\bar{P}(n)=\sum_{i=1}^{n} \pi\left(\hat{r}_{i}\right)$.

Proof of Proposition 3. To support the longest possible delay, the principal must receive her highest possible payoff $(\bar{P}(n))$ at the end of the delay, while she must receive the worst possible payoff if she deviates. Hence, the $T$-equilibrium that exhibits the longest delay is supported by the following strategies:

(1) The principal does not extend any offer for periods $0, \ldots, T-1$, where $T$ is the largest integer $t$ such that $\delta^{t} \bar{P}(n) \geqslant \underline{P}(n)$. 
(2) If there are no deviations from the prescription in (1), the low-offer equilibrium is implemented at date $T$.

(3) If the principal deviates at any time during (1), a standard-offer equilibrium is implemented immediately thereafter.

It is easy to see that specifications (1)-(3) constitute an equilibrium. The proposition now follows from the definition of $T$.

Proof of Proposition 4. Proposition 3 tells us that if there are $m$ free agents left in the game, the longest delay that can be endured is given by the largest integer $T(m)$ satisfying the inequality

$$
\delta^{T(m)} \geqslant \underline{P}(m) / \bar{P}(m) .
$$

Moreover, by an iterative argument, it is obvious that if a package $\mathbf{c}$ of $k$ offers are made and accepted at the end of this period, where $k \leqslant m$, then

$$
c_{i} \leqslant r_{m-k+i},
$$

where the $c_{i}$ 's have been arranged in non-decreasing order.

With these two observations in mind, consider any equilibrium, in which at dates $\tau_{1}, \tau_{2}$, $\ldots, \tau_{S}$, accepted offers are made. Let $n_{j}$ be the number of such accepted offers at date $\tau_{j}$; then, because no equilibrium permits infinite delay, we know that $\sum_{j=1}^{S} n_{j}=n$. Define $m_{1} \equiv n$ and recursively, $m_{j+1} \equiv m_{j}-n_{j}$ for $j=1, \ldots, S-1$; this is then the number of free agents left at the start of "stage $j$ ".

Set $\tau_{0} \equiv 0$. Notice that for every stage $j \geqslant 1$, that $\tau_{j}-\tau_{j-1} \leqslant T\left(m_{j}\right)$, so that by repeated use of (16), we may conclude that

$$
\delta^{\tau_{j}-\tau_{j-1}} \geqslant \delta^{T\left(m_{j}\right)} \geqslant \underline{P}\left(m_{j}\right) / \bar{P}\left(m_{j}\right)
$$

for all $j \geqslant 1$. Expanding (18), we may conclude that for all $j$,

$$
\delta^{\tau_{j}} \geqslant \prod_{k=1}^{j}\left[\underline{P}\left(m_{k}\right) / \bar{P}\left(m_{k}\right)\right] .
$$

Now denote by $a^{j}$ the average equilibrium payoffs to agents who close a deal at stage $j$ (date $\tau_{j}$ ). Then, using (17), we must conclude that

$$
a^{j} \leqslant\left(1-\delta^{\tau_{j}}\right) r_{n}+\delta^{\tau_{j}} \frac{1}{n_{j}} \sum_{i=1}^{n_{j}} r_{m_{j}-n_{j}+i},
$$

so that the overall average $a$-satisfies the inequality

$$
\begin{aligned}
a & \leqslant \frac{1}{n} \sum_{j=1}^{S}\left[\left(1-\delta^{\tau_{j}}\right) r_{n}+\delta^{\tau_{j}} \frac{1}{n_{j}} \sum_{i=1}^{n_{j}} r_{m_{j}-n_{j}+i}\right] n_{j} \\
& =r_{n}-\frac{1}{n} \sum_{j=1}^{S} \delta^{\tau_{j}}\left[r_{n}-\frac{1}{n_{j}} \sum_{i=1}^{n_{j}} r_{m_{j}-n_{j}+i}\right] n_{j}
\end{aligned}
$$




$$
\leqslant r_{n}-\frac{1}{n} \sum_{j=1}^{S} \prod_{k=1}^{j}\left[\underline{P}\left(m_{k}\right) / \bar{P}\left(m_{k}\right)\right]\left[r_{n}-\frac{1}{n_{j}} \sum_{i=1}^{n_{j}} r_{m_{j}-n_{j}+i}\right] n_{j},
$$

where the last inequality invokes (19).

Now all that remains to be seen is that the expression

$$
\frac{1}{n} \sum_{j=1}^{S} \prod_{k=1}^{j}\left[\underline{P}\left(m_{k}\right) / \bar{P}\left(m_{k}\right)\right]\left[r_{n}-\frac{1}{n_{j}} \sum_{i=1}^{n_{j}} r_{m_{j}-n_{j}+i}\right] n_{j}
$$

is strictly positive everywhere and bounded away from zero uniformly in $\delta$. The result follows.

\section{Acknowledgments}

We thank Jean-Pierre Benoît, Eric Maskin and Andrew Postlewaite for useful discussions, and Chinua Achebe for inspiring the title. We are grateful for comments by seminar participants at Ecares, Georgetown University, Penn State University, Cornell University, the South West Economic Theory conference at UCLA, and Yale University. Genicot gratefully acknowledges support under a Research and Writing grant from the John D. and Catherine T. MacArthur Foundation, and Ray acknowledges support from the National Science Foundation Grant No. 0241070.

\section{References}

[1] M. Balen, A Very English Deceit: The Secret History of the South Sea Bubble and the First Great Financial Scandal, Fourth Estate, 2003.

[2] P.K. Bardhan, On the concept of power in economics, Econ. Politics 3 (1991) 265-277.

[3] K. Basu, One kind of power, Oxford Econ. Pap. 38 (1986) 259-282.

[4] D. Bó, Bribing voters, Institute of Economics and Statistics Oxford, Discussion Papers 39, 2003.

[5] H. Cai, Delay in multilateral bargaining under complete information, J. Econ. Theory 93 (1999) 260-276.

[6] D. Gale, Dynamic coordination games, Econ. Theory 5 (1995) 103-143.

[7] G. Genicot, Bonded labor and serfdom: a paradox of voluntary choice, J. Devel. Econ. 67 (2002) 101-127.

[8] A. Gomes, P. Jehiel, Dynamic processes of social and economic interactions: on the persistence of inefficiencies, J. Polit. Economy 113 (2005) 626-667.

[9] S.J. Grossman, O. Hart, Takeover bids, the free-rider problem and the theory of the corporation, Bell J. Econ. (1981) 42-64.

[10] O. Hart, J. Tirole, Contract renegotiation and coasian dynamics, Rev. Econ. Stud. 55 (1988) 509-540.

[11] J. Hirshleifer, Paradox of power, Econ. Politics 3 (1991) 177-200.

[12] R. Innes, R. Sexton, Strategic buyers and exclusionary contracts, Amer. Econ. Rev. 84 (1994) 566-584.

[13] P. Jehiel, B. Moldovanu, Negative externalities may cause delay in negotiation, Econometrica 63 (1995) 1321-1335.

[14] P. Jehiel, B. Moldovanu, Cyclical delay in bargaining with externalities, Rev. Econ. Stud. 62 (1995) 619-637.

[15] J.J. Laffont, P. Rey, J. Tirole, Network competition: I. overview and nondiscriminatory pricing, II: price discrimination, RAND J. Econ. 29 (1998) 1-56.

[16] L. Makowski, Perfect competition, the profit criterion and the organization of economic activity, J. Econ. Theory 22 (1980) 222-242. 
[17] R.P. McAfee, M. Schwartz, Opportunism in multilateral vertical contracting: nondiscrimination, exclusivity, and uniformity, Amer. Econ. Rev. 84 (1994) 210-230.

[18] M. Moller, Sequential contracting with externalities, mimeo, 2004.

[19] M. Olasky, Fighting for Liberty and Virtue: Political and Cultural Wars in Eighteenth Century, Crossway Books, Wheaton III, 1995.

[20] E.B. Rasmusen, J.M. Rasmeyer, J.S. Wiley Jr., Naked exclusion, Amer. Econ. Rev. 81 (1991) 1137-1144.

[21] D. Ray, R. Vohra, A theory of endogenous coalition structure, Games Econ. Behav. 26 (1999) 286-336.

[22] I. Segal, Contracting with externalities, Quart. J. Econ. 114 (1999) 337-388.

[23] I. Segal, Coordination and discrimination in contracting with externalities: divide and conquer, J. Econ. Theory 113 (2003) 147-181.

[24] I. Segal, M. Whinston, Naked exclusion: comment, Amer. Econ. Rev. 90 (2000) 296-309.

[25] I. Segal, M. Whinston, Naked exclusion and buyer coordination, mimeo, Harvard Institute of Economic Research Working Papers 1780, 1996.

[26] B. Caillaud, B. Julien, Competing in network industries: Divide and conquer, RAND J. Econ. 34 (2003) $309-328$. 